\title{
SIMULTANEOUS APPROXIMATION OF ADDITIVE FORMS
}

\author{
BY
}

\author{
MING-CHIT LIU
}

\begin{abstract}
Let $X=\left(x_{1}, \cdots, x_{s}\right)$ be a vector of $s$ real components and $f_{i}(X)=\Sigma_{j=1}^{s} \theta_{i j} x_{j}^{k}(k=2,3, \cdots ; i=1, \cdots, R) R$ additive forms, where $\theta_{i j}$ are arbitrary real numbers. The author obtains some results on the simultaneous approximation of $\left\|f_{i}(X)\right\|$, where $\|t\|$ means the distance from $t$ to the nearest integer.
\end{abstract}

1. Introduction. In 1948 Heilbronn [5] improved Vinogradov's result [9] and obtained that for any $\epsilon>0$ there exists some positive constant $C=C(\epsilon)$ which depends on $\epsilon$ only such that for any real number $\theta$ and any integer $N \geqslant 1$ there is an integer $x$ satisfying

$$
1 \leqslant x \leqslant N \text { and }\left\|\theta x^{2}\right\|<C N^{-1 / 2+\epsilon},
$$

where $\|t\|$ means the distance from $t$ to the nearest integer.

In 1967, see [4], Davenport generalized (1.1) and obtained

$$
1 \leqslant x \leqslant N \text { and }\left\|\theta x^{k}\right\|<C N^{-(1 / K)+\epsilon},
$$

where $K=2^{k-1}, k=2,3, \cdots$ and $C=C(\epsilon, k)$ is a positive constant depending on $\epsilon, k$ only. Recently, R. J. Cook [2] extended (1.2) to a finite number of $\theta$ 's. On the other hand, Cook [3] and the author [8] obtained some results on additive forms. These results are as follows:

THEOREM C [2]. For any $\epsilon>0$ and any integers $k>1, R>0$, there exists a positive constant $C=C(\epsilon, k, R)$ depending on $\epsilon, k, R$ only such that for any real numbers $\theta_{1}, \cdots, \theta_{R}$ and any integer $N \geqslant 1$ there is an integer $x$ satisfying

$$
1 \leqslant x \leqslant N \text { and } \max _{1 \leqslant i<R}\left(\left\|\theta_{i} x^{k}\right\|\right)<C N^{-(1 / f(k, R))+\epsilon},
$$

where $f(k, R)$ is defined by $K=2^{k-1}$ and

$$
f(k, 1)=K, \quad f(k, R)=2 f(k, R-1)+K R+1 \quad(R \geqslant 2) .
$$

THEOREM L [8]. For any integers $k \geqslant 2$ and $s \geqslant 1$, put $K=2^{k-1}$ and $X=$ $\left(x_{1}, \cdots, x_{s}\right), a$ vector of $s$ real components. Let $f(X), g(X)$ be any two additive forms of degree $k$ in $s$ variables,

Received by the editors October 25, 1973 .

AMS(MOS) subject classifications (1970). Primary 10F10; Secondary $10 \mathrm{C} 99$.

Key words and phrases. Additive form, Diophantine approximation, simultaneous approximation, asymptotic behaviour. 


$$
f(X)=\sum_{i=1}^{s} \theta_{i} x_{i}^{k}, \quad g(X)=\sum_{i=1}^{s} \phi_{i} x_{i}^{k},
$$

where $\theta_{1}, \cdots, \theta_{s} ; \phi_{1}, \cdots, \phi_{s}$ are arbitrary real numbers. Then for any $\epsilon>0$ and any integer $N \geqslant 1$ there are integers $x_{1}, \cdots, x_{s}$ not all zero and some positive constant $C=C(\epsilon, k, s)$ which depends on $\epsilon, k$, s only such that

$$
\begin{gathered}
1 \leqslant \max _{1 \leqslant i \leqslant s}\left|x_{i}\right| \leqslant N, \\
\max (\|f(X)\|,\|g(X)\|)<C N^{-(1 / F(k, s))+\epsilon,}
\end{gathered}
$$

where

$$
F(k, s)= \begin{cases}7 & \text { if } s=1, k=2, \\ 3 K+1 / k & \text { if } s=1, k \geqslant 3, \\ 2 K+1+K / k s & \text { if } s \geqslant 2 .\end{cases}
$$

In this paper we shall extend Theorem $L$ to a finite number of additive forms with some improvements of Theorem $\mathrm{C}$. We shall prove

THEOREM 1. For any integers $k \geqslant 2$ and $s \geqslant 1$, put $K=2^{k-1}$ and $X=$ $\left(x_{1}, \cdots, x_{s}\right)$, a vector of $s$ real components. For any integer $R \geqslant 3$ let

$$
f_{i}(X)=\sum_{j=1}^{s} \theta_{i j} x_{j}^{k} \quad(i=1, \cdots, R)
$$

be any $R$ additive forms of degree $k$ in $s$ variables, where $\theta_{i j}(i=1, \cdots, R ; j=$ $1, \cdots, s)$ are arbitrary real numbers. Then for any $\epsilon>0$ and any integer $N \geqslant 1$ there are integers $x_{1}, \cdots, x_{s}$ not all zero and some positive constant $C=$ $C(\epsilon, k, s, R)$ which depends on $\epsilon, k, s, R$ only such that

$$
\begin{gathered}
1 \leqslant \max _{1<j<s}\left|x_{j}\right| \leqslant N, \\
\max _{1 \leqslant i \leqslant R}\left(\left\|f_{i}(X)\right\|\right)<C N^{-(1 / G(k, s, R))+\epsilon,}
\end{gathered}
$$

where $G(k, s, R)$ is defined by

$$
G(k, s, R)=2 g(k, R-1)+(R-1) K / k s+1 \quad(R \geqslant 3)
$$

and $g(k, R)$ is defined by

$$
\begin{aligned}
& g(k, 2)= \begin{cases}7 & \text { if } k=2, \\
3 K+1 / k & \text { if } k \geqslant 3 .\end{cases} \\
& g(k, R)=2 g(k, R-1)+(R-1) K / k+1 \quad(R \geqslant 3) .
\end{aligned}
$$

COROLlaRY 1. For any $\epsilon>0$ and any integers $k>1, R>1$ there exists a positive constant $C=C(\epsilon, k, R)$ depending on $\epsilon, k, R$ only such that for any 
real numbers $\theta_{1}, \cdots, \theta_{R}$ and any integer $N \geqslant 1$ there is an integer $x$ satisfying

$$
1 \leqslant x \leqslant N \text { and } \max _{1 \leqslant i \leqslant R}\left(\left\|\theta_{i} x^{k}\right\|\right)<C N^{-(1 / g(k, R))+\epsilon} \text {, }
$$

where $g(k, R)$ is defined by (1.9).

Corollary 1 follows from Theorem $\mathrm{L}(R=2)$ and Theorem $1(R \geqslant 3)$.

We shall give the explicit form of $g(k, R)$ in Lemma $1((2.1),(2.2))$ for the sake of application. For example when $k \geqslant 3$ we have:

\begin{tabular}{|c|l|}
\hline$R$ & $g(k, R)$ \\
\hline 2 & $3 K+1 / k$ \\
\hline 3 & $(6+2 / k) K+1+2 / k$ \\
\hline 4 & $(12+7 / k) K+3+4 / k$ \\
\hline 5 & $(24+18 / k) K+7+8 / k$ \\
\hline$\ldots \ldots \ldots$ & $\ldots$ \\
\hline
\end{tabular}

I wish to thank the referee for his very helpful suggestions which brought improvements on the presentation of this paper.

2. Notation and preliminary lemmas. In what follows $k, s, R$ are the integers given in Theorem 1. We always assume that $\epsilon$ is a small enough positive quantity which is not the same $\epsilon$ given in Theorem 1 and that $N$ is a sufficiently large positive integer, say $N>N_{0}=N_{0}(\epsilon, k, s, R)$ which is a positive integer depending on $\epsilon, k, s, R$ given, such that all the subsequent asymptotic approximations and inequalities in $\S \S 3,4$ are satisfied. So it is difficult to define $\epsilon$ and $N_{0}$ at the beginning or at any particular point. If $y>0$ we use $x \ll y$ to denote $|x|<C y$, where $C$ is some positive constant which can depend on $\epsilon, k, s, R$. [t] is the integral part of $t$. For real $t$ we write $e(t)=\exp (2 \pi t i)$. We shall use $B$ to denote some irrelevant numbers which need not be the same from one occurrence to another. For figures $x, y,(x)_{y}$ indicates the $y$ th formula in $(x)$.

We need several lemmas.

LEMMA 1. Let $k, R$ be positive integers and $K=2^{k-1}$. If

where

$$
g(k, R)=2 g(k, R-1)+K(R-1) / k+1 \quad(R \geqslant 3),
$$

$$
g(k, 2)= \begin{cases}7 & \text { if } k=2, \\ 3 K+1 / k & \text { if } k \geqslant 3,\end{cases}
$$


then

(2.2) $g(k, R)=\left(3 K+\frac{3 K+k+1}{k}\right) 2^{R-2}-K\left(\frac{R+1}{k}+\frac{1}{K}\right)$ if $k \geqslant 3$.

Proof. Since for $R \geqslant 3, k \geqslant 2$ we have

$$
g(k, R)=2^{R-2} g(k, 2)+\sum_{i=0}^{R-3} 2^{i}+\frac{K}{2 k} \sum_{i=1}^{R-2}(R-i)\left(2^{i}\right),
$$

then (2.1), (2.2) follow by a simple calculation.

LEMMA 2. Suppose that $\Delta$ satisfies $0<\Delta<1 / 2$ and $r$ is a positive integer. Then there exists a real valued function $\psi(x)$, periodic with period 1 , which satisfies

$$
\psi(x)=0 \text { if }\|x\|>\Delta,
$$

$$
\psi(x)=\sum_{u=-\infty}^{\infty} c_{u} e(u x)
$$

where $c_{u}$ are real and

$$
c_{0}=\Delta, \quad\left|c_{u}\right|<C(r) \min \left(\Delta, \Delta^{-r}|u|^{-r-1}\right) \text { if } u \neq 0,
$$

where $C(r)$ is some positive constant depending on $r$ only.

Proof. This is a particular case of Lemma 12 in $[10$, p. 32] with $\beta=-\alpha=$ $1 / 2 \Delta$.

Lemma 3. Let $T=\Sigma_{x=1}^{N} e\left(t x^{k}\right)$, where $k=2,3, \cdots$ and $t$ is any real number. For any $\epsilon>0$ we have

$$
|T|^{K} \ll N^{K-1}+N^{K-k+\epsilon} \sum_{j=1}^{L} \min (N, 1 /\|j t\|),
$$

where $K=2^{k-1}$ and $L=k ! N^{k-1}$.

Proof. See Satz 266 in [7, p. 255].

LEMMA 4. For any real $t$ if there are integers $a, q$ with $q>0$ such that $(a, q)=1$ and $|t-a / q| \leqslant q^{-2}$, then for any positive integers $P$ and $N$,

$$
\sum_{j=P+1}^{P+q} \min (N, 1 /\|j t\|) \ll N+q \log q .
$$

Proof. Lemma 4 is well known. See, for example, Lemma 3.5 in [6].

Lemma 5. For any integer $k \geqslant 2$ put $K=2^{k-1}$. For any $\epsilon$ with $0<\epsilon<1$ and any integers $s \geqslant 1, R \geqslant 3$ let $\lambda$ satisfy 


$$
\lambda\{2 g(k, R-1)+(R-1) K / s k+1\}<1-\epsilon
$$

and

$$
\alpha=\frac{2}{K} g(k, R-1)+\frac{(R-1)(1-k)}{s k}+\frac{1}{K},
$$

where $g(k, R)$ is defined by (1.9). Then the following five inequalities hold simultaneously.

$$
\begin{gathered}
(\lambda / s) R K<1-\epsilon, \\
(N s)(s K \alpha+(R-1) K)<1-\epsilon, \\
(\lambda(k-1) s)\left(K\left(1+R^{2}\right)+s R-s \alpha K\right)<1-\epsilon, \\
(\lambda(k-1))(2 k g(k, R-1)-\alpha K+k)<1-\epsilon, \\
\lambda(2 g(k, R-1)+1)<1-\epsilon .
\end{gathered}
$$

Proof. By (1.9) it is easy to see that $g(k, R-1)>K R(R=3,4, \cdots)$. It follows that $(2.7) \Rightarrow(2.13) \Rightarrow(2.9)$. On the other hand, substituting $(2.8)$ into $(2.10)$ and $(2.12)$ we see that $(2.7)=(2.10)=(2.12)$. So it remains to show that when $R \geqslant 3$

$$
2 k g(k, R-1)+k>K\left(1+R^{2}\right)+R,
$$

i.e. (2.12) $\Rightarrow(2.11)$. Here we only give the arguments for $k \geqslant 3$. By (2.2) we have

$$
2 k g(k, R-1)+k=3 K\left(2^{R-2}\right)(k+1)+k\left(2^{R-2}-1\right)+2^{R-2}-2 K R .
$$

Then (2.14) follows since when $k \geqslant 3, R \geqslant 3$ we have

$$
3\left(2^{R-2}\right)(k+1)>(1+R)^{2} \text { and } k\left(2^{R-2}-1\right)+2^{R-2}>R .
$$

The proof of Lemma 5 is complete.

LEMMA 6. Let $R, N$ be any positive integers and $\theta_{1}, \cdots, \theta_{R}$ any real numbers. Then there exists an integer $n$ satisfying

$$
1 \leqslant n \leqslant N, \quad\left\|\theta_{i} n\right\|<N^{-1 / R} \quad(i=1, \cdots, R) .
$$

Proof. See Theorem VI [1, p. 13].

3. Existence of an $R$-tuple. We come now to the proof of our theorem.

Suppose that for some $\lambda>0$ there are no integral solutions $X=\left(x_{1}, \cdots\right.$, $x_{s}$ ) of the following inequalities:

$$
1 \leqslant \max _{1 \leqslant j \leqslant s}\left|x_{j}\right| \leqslant N, \quad \max _{1 \leqslant i \leqslant R}\left(\left\|f_{i}(X)\right\|\right) \leqslant N^{-\lambda},
$$


i.e. for each $X$ with integers $x_{1}, \cdots, x_{s}$ satisfying $1 \leqslant \max _{1 \leqslant j \leqslant s}\left|x_{j}\right| \leqslant N$, we have some $i(1 \leqslant i \leqslant R)$ such that $\left\|f_{i}(X)\right\|>N^{-\lambda}$. Putting $\Delta=N^{-\lambda}$ in Lemma 2 , we have

$$
\begin{aligned}
0 & =\sum_{X} \psi\left(f_{1}(X)\right) \cdots \psi\left(f_{R}(X)\right) \\
& =\sum_{X}\left\{\sum_{m_{1}=-\infty}^{\infty} c_{m_{1}} e\left(m_{1} f_{1}(X)\right) \cdots \sum_{m_{R}=-\infty}^{\infty} c_{m_{R}} e\left(m_{R} f_{R}(X)\right)\right\} \\
& =N^{s} c_{0}^{R}+\sum_{X} \sum_{\mathrm{m}} c_{m_{1}} \cdots c_{m_{R}} e\left(m_{1} \sum_{j=1}^{s} \theta_{1 j} x_{j}^{k}\right) \cdots e\left(m_{R} \sum_{j=1}^{s} \theta_{R j} x_{j}^{k}\right),
\end{aligned}
$$

where $\Sigma_{X}$ is taken over integers $x_{j}$ with $1 \leqslant j \leqslant s, 1 \leqslant x_{j} \leqslant N$, and $\Sigma_{\mathrm{m}}$ is taken over $-\infty<m_{1}, \cdots, m_{R}<\infty$ except $\mathrm{m}=\left(m_{1}, \cdots, m_{R}\right)=(0, \cdots, 0)$. By (2.4) with $c_{0}=N^{-\lambda}$ and (3.2) we have

$$
N^{s-R \lambda} \leqslant \sum_{\mathrm{m}}\left|c_{m_{1}} \cdots c_{m_{R}}\right| \prod_{j=1}^{s}|S(\mathrm{~m}, j)|
$$

where

$$
S(\mathrm{~m}, j)=\sum_{x=1}^{N} e\left(x^{k} \sum_{i=1}^{R} \theta_{i j} m_{i}\right) \quad(j=1, \cdots, s) .
$$

Write

$$
\sum_{\mathrm{m}}\left|c_{m_{1}} \cdots c_{m_{R}}\right| \prod_{j=1}^{s}|S(\mathrm{~m}, j)|=\left(\sum_{1}+\sum_{2}\right)\left|c_{m_{1}} \cdots c_{m_{R}}\right| \prod_{j=1}^{s}|S(\mathrm{~m}, j)|,
$$

where $\Sigma_{1}$ is the summation taken over $\left|m_{i}\right|<N^{\lambda+\epsilon}(i=1, \cdots, R)$ and $\mathrm{m} \neq$ $(0, \cdots, 0)$ while $\Sigma_{2}$ is taken over all remaining terms in $\Sigma_{\mathrm{m}}$. We are going to show that $\Sigma_{2} \ll N^{s-R \lambda-\epsilon}$ if we let $r$ given in Lemma 2 satisfy $r>R(\lambda+\epsilon) / \epsilon$. So in view of (3.3), (3.5) we may neglect $\Sigma_{2}$.

For each $l=1, \cdots, R$ by $(2.4)_{2}$ we have

$$
\sum_{\left|m_{l}\right|<\infty}\left|c_{m_{l}}\right|=\sum_{\left|m_{l}\right|<N^{\lambda+\epsilon}}\left|c_{m_{l}}\right|+\sum_{\left|m_{l}\right| \geqslant N \lambda+\epsilon}\left|c_{m_{l}}\right|
$$

$$
\begin{aligned}
& \ll N^{-\lambda} \sum_{\left|m_{l}\right|<N^{\lambda+\epsilon}} 1+N^{r \lambda} \sum_{\left|m_{l}\right| \geqslant N^{\lambda+\epsilon}}\left|m_{l}\right|^{-r-1} \\
& \ll N^{\epsilon}+N^{r \lambda} N^{-r(\lambda+\epsilon)} \ll N^{\epsilon} .
\end{aligned}
$$

Since by (3.4), $|S(\mathrm{~m}, j)| \leqslant N$ we see that 


$$
\begin{aligned}
\sum_{2} & \leqslant \sum_{i=1}^{R}\left\{\sum_{\left|m_{i}\right| \geqslant N^{\lambda+\epsilon}}\left|c_{m_{1}} \cdots c_{m_{R}}\right| \prod_{j=1}^{s}|S(\mathrm{~m}, j)|\right\} \\
& \leqslant N^{s} \sum_{i=1}^{R}\left\{\sum_{\left|m_{i}\right| \geqslant N \lambda+\epsilon}\left|c_{m_{i}}\right|\right\}\left\{\prod_{i \neq l} \sum_{\left|m_{l}\right|<\infty}\left|c_{m_{l}}\right|\right\} .
\end{aligned}
$$

By (3.6) we have

$$
\begin{aligned}
\sum_{2} & \ll N^{s} \sum_{i=1}^{R}\left\{\sum_{\left|m_{i}\right| \geqslant N^{\lambda+\epsilon}}\left|c_{m_{i}}\right|\right\} N^{(R-1) \epsilon} \\
& \ll N^{s+(R-1) \epsilon} \sum_{i=1}^{R} N^{-r \epsilon} \ll N^{s+(R-1) \epsilon-r \epsilon} \ll N^{s-R \lambda-\epsilon},
\end{aligned}
$$

if $r>R(\lambda+\epsilon) / \epsilon$.

It follows from (3.3), (3.5) (2.4) $)_{2}$ that

$$
\begin{aligned}
N^{s-R \lambda} & \ll \sum_{1}\left|c_{m_{1}} \cdots c_{m_{R}}\right| \prod_{j=1}^{s}|S(\mathrm{~m}, j)| \\
& \ll \sum_{1} N^{-R \lambda} \prod_{j=1}^{s}|S(\mathrm{~m}, j)| .
\end{aligned}
$$

That is

$$
N^{s} \ll \sum_{1} \prod_{j=1}^{s}|S(\mathrm{~m}, j)| \ll \sum_{j=1}^{s} \sum_{1}|S(\mathrm{~m}, j)|^{s} .
$$

We see that there exists some $j_{0}\left(1 \leqslant j_{0} \leqslant s\right)$ for which we write $\theta_{i}=\theta_{i j_{0}}$ and $S(\mathrm{~m})=S\left(\mathrm{~m}, j_{0}\right)$, such that

where, by (3.4),

$$
N^{s} \ll \sum_{1}|S(\mathrm{~m})|^{s},
$$

$$
S(\mathrm{~m})=\sum_{x=1}^{N} e\left(x^{k} \sum_{i=1}^{R} m_{i} \theta_{i}\right) .
$$

By definition of the notation $\ll$ there is some positive constant $B$ such that we may rewrite (3.8) as

$$
B N^{s} \leqslant \sum_{1}|S(\mathrm{~m})|^{s}
$$

We are now going to show that by (3.8a) there exists a $\rho$

$$
0 \leqslant \rho \leqslant \lambda+\epsilon
$$




$$
\begin{aligned}
& |S(\mathrm{~m})| \geqslant\left(2^{-R-1} B\right)^{1 / s} N^{1-R p} \quad\left(N^{1-R p}\right), \\
& p=\rho / s,
\end{aligned}
$$

for at least $\left[N^{R \rho-\epsilon}\right]+1 R$-tuples $\left(m_{1}, \cdots, m_{R}\right)$ in

$$
\left|m_{i}\right|<N^{\lambda+\epsilon} \quad(i=1, \cdots, R) .
$$

Suppose that such a $\rho$ does not exist. For some integer $l$ with $l \epsilon>2 R(\lambda+\epsilon)$ write

$$
\sum_{1}|S(\mathrm{~m})|^{s}=\sum_{3}|S(\mathrm{~m})|^{s}+\sum_{j=0}^{l-1} T_{j}
$$

where $\Sigma_{3}$ is taken over $\left|m_{i}\right|<N^{\lambda+\epsilon}(i=1, \cdots, R), \mathrm{m} \neq(0, \cdots, 0)$ and $|S(\mathrm{~m})|^{s}<2^{-R-1} B N^{s-R(\lambda+\epsilon)}$ while $T_{j}=\Sigma|S(\mathrm{~m})|^{s}$ which are taken over $\left|m_{i}\right|<$ $N^{\lambda+\epsilon}(i=1, \cdots, R), \mathrm{m} \neq(0, \cdots, 0)$ and

$$
2^{-R-1} B N^{(s-(j+1) R(\lambda+\epsilon) / l)} \leqslant|S(\mathrm{~m})|^{s}<2^{-R-1} B N^{(s-j R(\lambda+\epsilon) / l)} .
$$

According to our assumption on $\rho$ there are no such terms $|S(\mathrm{~m})|^{s}$ satisfying $2^{-R-1} B N^{s} \leqslant$ $|S(\mathrm{~m})|^{s}$ even if $2^{-R-1} B<1$. So we neglect this possibility in the above summation $\Sigma_{j=0}^{l-1} T_{j}$. Now by our supposition on $\rho$ and $\epsilon / 2>R(\lambda+\epsilon) / l$ we have

$$
\begin{aligned}
T_{j} \leqslant\left(2^{-R-1} B N^{(s-j R(\lambda+\epsilon) / l)}\right)\left(N^{((j+1) R(\lambda+\epsilon) / l-\epsilon)}\right) & \\
= & 2^{-R-1} B N^{(s-\epsilon+R(\lambda+\epsilon) / l)}<2^{-R-1} B N^{s-\epsilon / 2} \quad(j=0,1, \cdots, l-1) . \\
& \sum_{3}|S(\mathrm{~m})|^{s}<\left(2^{-R-1} B N^{s-R(\lambda+\epsilon)}\right)\left(2 N^{\lambda+\epsilon}\right)^{R}=B N^{s} / 2 .
\end{aligned}
$$

Hence

$$
\begin{aligned}
\sum_{1}|S(\mathrm{~m})|^{s} & =\sum_{3}|S(\mathrm{~m})|^{s}+\sum_{j=0}^{l-1} T_{j} \\
& <\left(B N^{s / 2}\right)+\left(2^{-R-1} B I N^{s-\epsilon / 2}\right)<B N^{s}
\end{aligned}
$$

if $N$ is large. This contradicts (3.8a). So a $\rho$ satisfying (3.10), (3.11) exists.

In what follows we shall confine our attention to the $R$-tuples $\left(m_{1}, \cdots\right.$; $m_{R}$ ) satisfying (3.10), (3.11), (3.12). From (2.5), (3.9) we have

where $L=k ! N^{k-1}$ and

$$
|S(\mathrm{~m})|^{K} \ll N^{K-1}+N^{K-k+\epsilon} \sum_{j=1}^{L} \min (N, 1 /\|j t\|),
$$

$$
t=\sum_{i=1}^{R} \theta_{i} m_{i}
$$

Define 


$$
Q=N^{k-1+\alpha \lambda K-K p} \quad(p=\rho / s)
$$

where

$$
\alpha=\alpha(k, s, R)=\frac{2}{K} g(k, R-1)+\frac{(R-1)(1-k)}{k s}+\frac{1}{K}
$$

and $g(k, R)$ is defined by (1.9). By Dirichlet's theorem for given $t$ and $Q((3.14)$, (3.15)) there are integers $a, q$ such that

$$
(a, q)=1, \quad 1 \leqslant q \leqslant Q, \quad|q t-a|<Q^{-1} .
$$

Fix such a $q$ and divide the sum in the right of (3.13) into blocks of $q$ terms. It follows from Lemma 4 and (3.13) that

$$
|S(\mathrm{~m})|^{K} \ll N^{K-1}+N^{K-k+\epsilon}\left(k ! N^{k-1} q^{-1}+1\right)(N+q \log q) .
$$

Then by (3.11) we have

$$
N^{1-R K p-\epsilon} \ll N q^{-1}+N^{\epsilon}+N^{1-k+\epsilon} q .
$$

Suppose that $\lambda$ satisfies

$$
\lambda\left\{2 g(k, R-1)+\frac{(R-1) K}{s k}+1\right\}<1-A \epsilon \quad(R \geqslant 3),
$$

where $g(k, R)$ is defined by (1.9) and $A$ is a positive constant. The value of $A$ is so defined such that all following inequalities (3.21), (3.22), (3.26), (4.8), (4.10) will be satisfied.

By (3.15), (3.17) $2,(3.18)$ we have

$$
q^{-1} \gg N^{-R K p-\epsilon}\left\{1-N^{2 \epsilon+R K p-1}-N^{R K p+2 \epsilon-1+\alpha \lambda K-K p}\right\} .
$$

The last two terms in the curly bracket of (3.20) can be neglected since by (3.10), (3.19) and Lemma 5 ((2.9), (2.10)) we have

$$
2 \epsilon+R K p-1<0
$$

and

$$
R K p+2 \epsilon-1+\alpha \lambda K-K p<0 .
$$

Then from (3.20) we have

$$
\left(1 \leqslant q \ll N^{R K p+\epsilon}\right. \text {. }
$$

By Lemma 6 , for given $\theta_{1}, \cdots, \theta_{R}$ and integer $\left[Q N^{-\epsilon}\right]$ there are integers $a_{1}, \cdots, a_{R}$ and $b$ such that

(3.24) $1 \leqslant b \leqslant\left[Q N^{-\epsilon}\right], \quad\left|b \theta_{i}-a_{i}\right|<\left[Q N^{-\epsilon} \Gamma^{-1 / R} \quad(i=1, \cdots, R)\right.$.

It follows from (3.12), (3.14), (3.17) 3 , (3.23) that 


$$
\begin{aligned}
\left|q \sum_{i=1}^{R} a_{i} m_{i}-a b\right| & \leqslant q \sum_{i=1}^{R}\left|m_{i}\right|\left|a_{i}-\theta_{i} b\right|+b\left|q\left(\sum_{i=1}^{R} \theta_{i} m_{i}\right)-a\right| \\
& \ll N^{R K p+\epsilon} \sum_{i=1}^{R} N^{\lambda+\epsilon}\left[Q N^{-\epsilon}\right]^{-1 / R}+\left[Q N^{-\epsilon}\right] Q^{-1} .
\end{aligned}
$$

By (3.10), (3.19) and Lemma 5 ((2.11)) we see that

$$
\begin{aligned}
& (R K p+\epsilon)+(\lambda+\epsilon)-\frac{1}{R}(k-1+\alpha \lambda K-K p-\epsilon) \\
& \quad<\frac{(k-1)}{R}\left\{\frac{\lambda}{s(k-1)}\left(K\left(1+R^{2}\right)+s R-s \alpha K\right)+1+B \epsilon\right\}<-\epsilon,
\end{aligned}
$$

where $B$ is some number depending on $k, s, R$ only. Then from (3.15), (3.25), (3.26) we have $\left|q \sum_{i=1}^{R} a_{i} m_{i}-a b\right| \ll N^{-\epsilon}<1$, for large $N$. That is $q \sum_{i=1}^{R} a_{i} m_{i}=$ $a b$. But by $(3.17)_{1}((a, q)=1)$ we see that $q$ divides $b$. Then by (3.15), (3.24) the number of possibilities for $q$ is $O\left(N^{\epsilon}\right)$. Since at least $\left[N^{R \rho-\epsilon}\right]+1(>1)$ $R$-tuples $\left(m_{1}, \cdots, m_{R}\right)$ in (3.12) satisfy (3.11) then $\gg N^{R \rho-2 \epsilon}$ (or $\geqslant B N^{R \rho-2 \epsilon}$ for some positive constant $B$ ) of these $R$-tuples have the same $q$. Choose a suitable $d\left(d^{R} B \geqslant 2^{R+1}\right.$, say) such that the following pigeonhole argument holds. Partition the $R$-cube, $\left|m_{i}\right| \leqslant N^{\lambda+\epsilon}(i=1, \cdots, R)$ by

$$
\left|m_{i}\right|=l d N^{\lambda+\epsilon-(\rho-(2 \epsilon / R))},
$$

where $i=1, \cdots, R ; l=0,1, \cdots$. In all, there are at most

$$
\left(\frac{2 N^{\lambda+\epsilon}}{d N^{\lambda+\epsilon-(\rho-(2 \epsilon / R))}}\right)^{R}=(2 / d)^{R} N^{R \rho-2 \epsilon}
$$

$R$-subcubes in the $R$-cube, $\left|m_{i}\right| \leqslant N^{\lambda+\epsilon}(i=1, \cdots, R)$. Now by the pigeonhole argument, there is an $R$-subcube containing at least two distinct $R$-tuples $\left(m_{1}^{\prime}\right.$, $\left.\cdots, m_{R}^{\prime}\right),\left(m_{1}^{\prime \prime}, \cdots, m_{R}^{\prime \prime}\right)$, say, having the same $q$. For these two $R$-tuples we may suppose that for some integer $I$ with $1 \leqslant I \leqslant R$ we have $m_{I}^{\prime}>m_{I}^{\prime \prime}$. Put

$$
m_{i}=m_{i}^{\prime}-m_{i}^{\prime \prime} \quad(i=1, \cdots, R) .
$$

In particular, we have

$$
m_{I} \geqslant 1 \text {. }
$$

Then by (3.27), (3.28) and (3.17) 3 we have

$$
\begin{aligned}
& \left|m_{i}\right| \ll N^{\lambda+\epsilon(1+(2 / R))-\rho} \quad(i=1, \cdots, R) . \\
& \left\|\left(\sum_{i=1}^{R} \theta_{i} m_{i}\right) q\right\| \leqslant\left\|\sum_{i=1}^{R} \theta_{i} m_{i}^{\prime} q\right\|_{i}+\left\|\sum_{i=1}^{R} \theta_{i} m_{i}^{\prime \prime} q\right\|<2 Q^{-1},
\end{aligned}
$$

since $R$-tuples $\left(m_{1}^{\prime}, \cdots, m_{R}^{\prime}\right),\left(m_{1}^{\prime \prime}, \cdots, m_{R}^{\prime \prime}\right)$ have the same $q$ in $(3.17)_{3}$. 
4. Completion of the proof. In what follows we shall confine our attention to the new $R$-tuple $\left(m_{1}, \cdots, m_{R}\right)$ satisfying (3.28), (3.30). We proceed by induction on $R$. As usual, our proof consists of two parts. We first show that Theorem 1 is true for $R(\geqslant 4)$ if we assume that Theorem 1 is true for $R-1$. Then we can see that in fact Theorem 1 holds for $R=3$.

Put

$$
\begin{gathered}
\phi_{i}=m_{I}^{k-1} q^{k} \theta_{i}, \\
M=\left[N^{\left(2 \lambda+2 \epsilon\left(1+R^{-1}\right)-\rho\right) g /(1-\epsilon g)}\right],
\end{gathered}
$$

where $g=g(k, R-1), i=1, \cdots ; R ; R \geqslant 3$. Suppose that Theorem 1 is true for $R-1(\geqslant 3)$. Then by Corollary $1,(1.10)$ is true for $M$ and any $R-1 \phi$ 's among $\phi_{1}, \cdots, \phi_{R}$. So there is some integer $n$ satisfying

$$
1 \leqslant n \leqslant M \text {, }
$$

$$
\max _{1 \leqslant i \leqslant R ; i \neq I}\left(\left\|\phi_{i} n^{k}\right\|\right) \ll M^{(-1 / g(k, R-1))+\epsilon} \ll N^{-2 \lambda-2 \epsilon\left(1+R^{-1}\right)+\rho} .
$$

Let

(4.4)

$$
x=n q m_{I} .
$$

It follows from (4.1), (4.3) $2,(3.30)_{1}$ that

$$
\left\|\theta_{i} x^{k}\right\| \leqslant\left\|\left(m_{I}^{k-1} q^{k} \theta_{i}\right) n^{k}\right\| m_{I}=\left\|\phi_{i} n^{k}\right\| m_{I} \quad(i=1, \cdots, R ; R \geqslant 4)
$$

and

$$
\max _{1 \leqslant i \leqslant R ; i \neq I}\left\|\theta_{i} x^{k}\right\| \ll N^{-2 \lambda-2 \epsilon\left(1+R^{-1}\right)+\rho} N^{\lambda+\epsilon(1+(2 / R))-\rho} \ll N^{-\lambda-\epsilon} .
$$

Similarly, we have

$$
\left\|\theta_{I} x^{k}\right\|=\left\|\theta_{I} n^{k} q^{k} m_{I}^{k}\right\|
$$

$$
\begin{aligned}
& \leqslant q^{k-1} n^{k} m_{I}^{k-1}\left\|q\left(\sum_{i=1}^{R} \theta_{i} m_{i}\right)\right\|+\sum_{i=1 ; i \neq I}^{R} \mid m_{i}\left\|\left(q^{k} m_{I}^{k-1} \theta_{i}\right) n^{k}\right\| \\
& \ll N^{\sigma_{1}}+N^{-\lambda-\epsilon},
\end{aligned}
$$

where $\sigma_{1}$ is defined (so as to make the last part of (4.6) valid) by the first part of (4.7). By (3.23), (4.2), (3.30), (3.15) we see that

$$
\begin{aligned}
\sigma_{1}= & (k-1)(R K p+\epsilon)+k\left(2 \lambda+2 \epsilon\left(1+R^{-1}\right)-\rho\right) g /(1-\epsilon g) \\
& +(k-1)(\lambda+\epsilon(1+(2 / R))-\rho)-(k-1+\alpha \lambda K-K p) \\
= & p((k-1) R K-s k g-(k-1) s+K) \\
& +\lambda(2 k g-\alpha K+k)+B \epsilon-(k-1)-\lambda-\epsilon,
\end{aligned}
$$


where $B$ depends on $k, R$ only. As pointed out in the proof of Lemma 5, $g(k, R-1)=g>K R$ when $R \geqslant 4$, whence we see that $(k-1) R K-s k g-(k-1) s+$ $K<0$. Then together with (4.7), (3.19), and Lemma 5 ((2.12)), we have

$$
\sigma_{1}<\lambda(2 k g(k, R-1)-\alpha K+k)-(k-1)+B \epsilon-\lambda-\epsilon<-\lambda-\epsilon .
$$

Hence by (4.5), (4.6), (4.8) we have

$$
\max _{1 \leqslant i \leqslant R}\left\|\theta_{i} x^{k}\right\| \ll N^{-\lambda-\epsilon} .
$$

Next, by (4.2), (3.23), (3.29), (3.30) 1 we see that $1 \leqslant n q m_{I}=x \ll N^{\sigma_{2}}$, where

$$
\begin{aligned}
\sigma_{2} & =\left(2 \lambda+2 \epsilon\left(1+R^{-1}\right)-\rho\right) g /(1-\epsilon g)+(R K p+\epsilon)+(\lambda+\epsilon(1+(2 / R))-\rho) \\
& =\rho(R K / s-g(k, R-1)-1)+\lambda(2 g(k, R-1)+1)+B \epsilon \\
& <\lambda(2 g(k, R-1)+1)+B \epsilon,
\end{aligned}
$$

for some $B$ depending on $k, R$ only. Then by (3.19) and Lemma 5 ((2.13)), we have

$$
\sigma_{2}<\lambda(2 g(k, R-1)+1)+B \epsilon<1-\epsilon
$$

Hence

$$
1 \leqslant x \ll N^{1-\epsilon} .
$$

(4.9) and (4.11) show that we have obtained an integer $x$ satisfying

$$
1 \leqslant x \leqslant N \text { and } \max _{1 \leqslant i \leqslant R}\left(\left\|\theta_{i} x^{k}\right\|\right) \leqslant N^{-\lambda} .
$$

This contradicts our supposition (3.1) since if we let $x_{j_{0}}=x$ (for $j_{0}$ see statement between (3.7) and (3.8)) and $x_{j}=0$ for all $j \neq j_{0}$ then we have a particular vector $X=\left(x_{1}, \cdots, x_{j}, \cdots, x_{s}\right)$ for which

$$
f_{i}(X)=\sum_{j=1}^{s} \theta_{i j} x_{j}^{k}=\theta_{i j_{0}} x_{j_{0}}^{k}=\theta_{i} x^{k},
$$

where $i=1, \cdots, R ; R \geqslant 4$. So by (3.19) with a suitable choice of $\epsilon$ Theorem 1 is true for $R(2)$, if it is true for $R-1$.

It remains to see that Theorem 1 is true for $R=3$. For the case $R=3$ the proof follows exactly in the same way as that for $R \geqslant 4$ except that now Corollary $1((1.10)$ ) is known for $R=2$ (a special case of Theorem $\mathrm{L}$ ). This proves Theorem 1 .

\section{REFERENCES}

1. J. W. S. Cassels, An introduction to Diophantine approximation, Cambridge Tracts in Math. and Math. Phys., no. 45, Cambridge Univ. Press, New York, 1957. MR 19, 396. 
2. R. J. Cook, On the fractional parts of a set of points, Mathematika 19 (1972), 63-68.

3. - The fractional parts of an additive form, Proc. Cambridge Philos. Soc. 72 (1972), 209-212. MR 45 \#6764.

4. H. Davenport, On a theorem of Heilbronn, Quart. J. Math. Oxford Ser. (2) 18 (1967), 339-344. MR 36 \#6355.

5. H. Heilbronn, On the distribution of the sequence $n^{2} \theta(\bmod 1)$, Quart. J. Math. Oxford Ser. 19 (1948), 249-256. MR 10, 284.

6. L. K. Hua, Additive theory of prime numbers, Trudy Mat. Inst. Steklov. 22 (1947); English transl., Transl. Math. Monographs, vol. 13, Amer. Math. Soc., Providence, R. I., 1965. MR 10, 597; 33 \#2614.

7. E. Landau, Vorlesungen über Zahlentheorie. Band I, Hirzel, Leipzig, 1927; English transl., Elementary number theory, Chelsea, New York, 1958. MR 19, 1159.

8. M. C. Liu, Simultaneous approximation of two additive forms, Proc. Cambridge Philos. Soc. 75 (1974), 77-82.

9. I. M. Vinogradov, Analytischer Beweis des Satzes über die Verteilung der Bruchteile eines ganzen Polynoms, Bull. Acad. Sci. USSR (6) 21 (1927), 567-578.

10. - The method of trigonometrical sums in the theory of numbers, Trudy Mat. Inst. Steklov. 23 (1947); English transl., Interscience, New York, 1954. MR 10, 599; 15,941 .

DEPARTMENT OF MATHEMATICS, UNIVERSITY OF HONG KONG, HONG KONG

Current address: Department of Pure Mathematics and Mathematical Statistics, University of Cambridge, Cambridge, England 